'Sección Endocrinología, Hospital Clínico de la Universidad de Chile. ${ }^{2}$ Hospital Regional de Copiapó. Copiapó, Chile.

${ }^{3}$ Laboratorio de Endocrinología y Biología de la Reproducción, Hospital Clínico de la Universidad de Chile. Santiago, Chile.

${ }^{4}$ Laboratorio de Nutrigenómica, Departamento de Nutrición, Facultad de Medicina, Universidad de Chile. 5IDIMI, Universidad de Chile. ${ }^{6}$ Unidad de Hemodinamia, Hospital San Borja-Arriarán ־Unidad de Endocrinología y Diabetes, Hospital San BorjaArriarán. Santiago, Chile. 'Tecnólogo Médico. bBioquímico. Interno de Medicina.

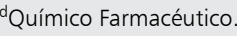

Conflictos de intereses: ninguno que declarar.

Recibido el 1 de marzo de 2015, aceptado el 7 de mayo de 2015.

Correspondencia a: Dr. Néstor Soto Isla. Amazonas 619, Santiago, Chile. Teléfono: 225748784 nsotoisla@gmail.com

\section{Síndrome de hipoglicemia autoinmune. Primeros casos en Chile}

\author{
ALEJANDRA LANAS ${ }^{1}$, ANA PAREDES ${ }^{2}$, CONSUELO ESPINOSA ${ }^{1}$, \\ EGARDO CAAMAÑO ${ }^{3, \mathrm{a}}$, FRANCISCO PÉREZ-BRAVO ${ }^{4, \mathrm{~b}}$, RODRIGO PINTO $^{1, \mathrm{c}}$, \\ GERMÁN IÑIGUEZ ${ }^{5, d}$, DARÍO MARTÍNEZ ${ }^{6}$, NÉSTOR SOTO ${ }^{7}$
}

\section{Insulin autoimmune syndrome. Report of two cases}

Insulin autoimmune syndrome (IAS) is characterized by spontaneous hypoglycemia with extremely high insulin levels and the presence of circulating autoantibodies against insulin, in patients who have never been exposed to exogenous insulin. We report two patients with the syndrome. A 36 years old male presenting with hypoglycemia in the emergency room had an oral glucose tolerance test showed basal and $120 \mathrm{~min}$ glucose levels of 88 and $185 \mathrm{mg} / \mathrm{dl}$. The basal and $120 \mathrm{~min}$ insulin levels were 2,759 and 5,942 $\mu \mathrm{UI} / \mathrm{ml}$. The presence of an insulin secreting tumor was discarded. Anti-insulin antibodies were positive. He was successfully treated with a diet restricted in carbohydrates and frequent meals in small quantities. A 65 years old female presenting with hypoglycemia in the emergency room had the fasting insulin levels of $1,910 \mu \mathrm{UI} / \mathrm{ml}$. No insulin secreting tumor was detected by images and anti-insulin antibodies were positive. The polyethylene glycol precipitation test showed a basal and after exposition insulin level 1,483 and $114 \mu \mathrm{UI} / \mathrm{ml}$, respectively. She responded partially to diet and acarbose and required the use of prednisone with a good clinical response.

(Rev Med Chile 2015; 143: 938-942)

Key words: Autoimmune response; Hypoglycemia; Insulin antibodies.

\begin{abstract}
1 síndrome de hipoglicemia autoinmune o Insulin autoimmune syndrome (IAS, enfermedad de Hirata) es una causa muy infrecuente de hipoglicemia. Se caracteriza por presentar hipoglicemia espontánea, niveles de insulina extremadamente elevados y presencia de autoanticuerpos circulantes contra la insulina en pacientes que nunca han recibido insulina exógena. Fue descrito en Japón ${ }^{1}$ y la mayoría de los casos han sido reportados en Asia, con muy escasos reportes en países occidentales. En Chile no se han comunicado casos en la literatura y nuestro objetivo es presentar los primeros dos casos.
\end{abstract}

\section{Caso 1}

Varón de 36 años, previamente sano, sin antecedentes familiares de diabetes, comenzó con epi- sodios de temblor, sudoración, debilidad de extremidades y síncope de seis meses de evolución que aparecían en ayunas, en relación con el ejercicio y cedían al comer. Estuvo en control neurológico por probable epilepsia, tratándose con carbamazepina algunas semanas, sin otros fármacos. Por un nuevo episodio de pérdida de conciencia consultó en Servicio de Urgencia pesquisándose hipoglicemia recurrente, la menor de $35 \mathrm{mg} / \mathrm{dl}$, siendo hospitalizado. El examen físico fue normal, sin acantosis nigricans y el índice de masa corporal de $25,6 \mathrm{~kg} /$ $\mathrm{m}^{2}$. La función tiroidea y niveles de cortisol matinal resultaron normales. Una prueba de tolerancia a la glucosa oral (PTGO) informó glicemia basal de $88 \mathrm{y}$ a los $120 \mathrm{~min} 185 \mathrm{mg} / \mathrm{dl}$, e insulinemia basal de 2.759 y a los $120 \mathrm{~min}$ de $5.942 \mu \mathrm{UI} / \mathrm{ml}$ (MEIA, Axym, Abbott). Se realizó test de ayuno presentando glicemia de $46 \mathrm{mg} / \mathrm{dl}$ e insulinemia de $588 \mu \mathrm{UI} /$ 
Tabla 1. Insulinemias $(\mu \mathrm{Ul} / \mathrm{ml})$ durante el test de estimulación selectiva con calcio en el caso 1

\begin{tabular}{|lccccc|}
\hline Arteria & Basal & $\mathbf{3 0}$ segundos & $\mathbf{6 0}$ segundos & $\mathbf{1 2 0}$ segundos & $\begin{array}{c}\text { Incremento } \\
\text { máximo }\end{array}$ \\
\hline Gastroduodenal & 659 & 891 & 897 & 688 & $36,1 \%$ \\
\hline Mesentérica superior & 698 & 918 & 1.109 & 1.042 & $58,9 \%$ \\
\hline Esplénica & 849 & 1.146 & 968 & 935 & $34,9 \%$ \\
\hline
\end{tabular}

ml (RIA, Diagnostic Products, Co [DPC]) a las 15 horas de ayuno, con sintomatología asociada. Una tomografía axial computada (TAC) de abdomen resultó normal. Se realizó un test de estimulación intraarterial selectiva con calcio para localización de insulinomas según protocolo ${ }^{2}$, el cual no permitió la localización (Tabla 1) y en la arteriografía se describió una posible lesión nodular en la cabeza del páncreas, de 1,7 x 1,4 cm, por lo que se realizó una laparatomía exploratoria que no demostró la presencia de tumor. Se midieron los anticuerpos antiinsulina mediante inmunoensayo enzimático (ELISA) con kits comerciales Medizym ${ }^{\circledR}$ (Medipan $\mathrm{GmbH}$, Berlín, Alemania) que resultaron positivos. Los anticuerpos antitiroideos y antinucleares fueron negativos. No presentó manifestaciones de otra enfermedad autoinmune.

Se trató con dieta restringida en carbohidratos refinados y comidas frecuentes en pequeñas cantidades, manteniéndose asintomático, sin presentar nuevas hipoglicemias. Al año de seguimiento se realizó PTGO que informó glicemia basal 84 y a los $120 \mathrm{~min} 240 \mathrm{mg} / \mathrm{dl}$ e insulinemia basal de 434 y a los $120 \mathrm{~min}$ de $793 \mu \mathrm{UI} / \mathrm{ml}$ (RIA). Se mantenía asintomático después de tres años de seguimiento.

\section{Caso 2}

Mujer de 65 años con antecedente de hipertensión arterial y depresión en tratamiento con fluoxetina y losartan. Presentó durante dos semanas episodios de sudoración, temblor y cefalea que ocurrieron predominantemente postprandiales tardíos. Posterior a un nuevo episodio asociado a compromiso de conciencia, ingresó al Servicio de Urgencia con glicemia de $43 \mathrm{mg} / \mathrm{dl}$, siendo hospitalizada. Negó el consumo de otros fármacos y no existían antecedentes familiares ni personales de diabetes mellitus. En el examen físico presentaba IMC de $29 \mathrm{~kg} / \mathrm{m}^{2}$, sin acantosis nigricans ni otros hallazgos. Dentro de los exámenes destacaban hormonas tiroideas normales, HbA1c 6,4\%, cortisol basal 11,7 $\mu \mathrm{g} / \mathrm{dl}$, insulinemia $2.910 \mu \mathrm{UI} / \mathrm{ml}$ (ECLIA Roche), péptido C 19,5 ng/ml, sulfonilureas en orina negativo. Se realizó test de ayunas sin presentar hipoglicemia, manteniendo niveles de insulina y péptidos $C$ persistentemente elevados. Se realizó PTGO que mostró hiperinsulinemia e hiperglicemia precoz con hipoglicemia posterior (Figura 1).

Se realizaron TAC y RNM de abdomen, endosonografía de páncreas y ${ }^{68} \mathrm{Ga}$ tomografía por emisión de positrones (PET) que resultaron normales. Se solicitaron anticuerpos anti insulina, que fueron positivos. Se determinó insulinemia basal y posterior a precipitación con polietilenglicol $(\mathrm{PEG})^{3}$ que fue de 1.483 y $114 \mu \mathrm{UI} / \mathrm{ml}$, respectivamente. Se diagnosticó además síndrome de Sjögren con Anti-Ro positivo. La cuantificación de inmunoglobulinas y electroforesis de proteínas fueron normales. Se determinó HLA clase II por PCR, utilizando el kit Dynal Reli que permitió analizar los haplogrupos DQ/DR resultando el haplotipo HLA DQ2-DQ8 y HLA-DR3-DR4. Posteriormente se utilizó un análisis extendido de alta resolución para los haplotipos específicos de las subregiones $\alpha$ y $\beta$ del complejo HLA de clase II detectándose el siguiente haplotipo: HLA-DQB1 ${ }^{\star} 0201-$ DQB1 ${ }^{\star} 0302$ y HLA-DQA $1^{\star}$ 0501 - DQA $1^{\star} 0301$.

Se trató con dieta fraccionada con bajo índice glicémico, presentando respuesta parcial. Se agregó acarbosa con buena respuesta inicial, pero por nuevos episodios de hipoglicemia se agregó prednisona $30 \mathrm{mg} /$ día. A los 2 meses de tratamiento se midió insulinemia basal de $462 \mu \mathrm{UI} / \mathrm{ml}$ y post PEG $46 \mu \mathrm{UI} / \mathrm{ml}$. Durante el seguimiento los síntomas e insulinemias descendieron de manera progresiva (Figura 2), permitiendo suspender la terapia farmacológica. Actualmente se encuentra asintomática, sin necesidad de terapia y con niveles de insulina en disminución. 

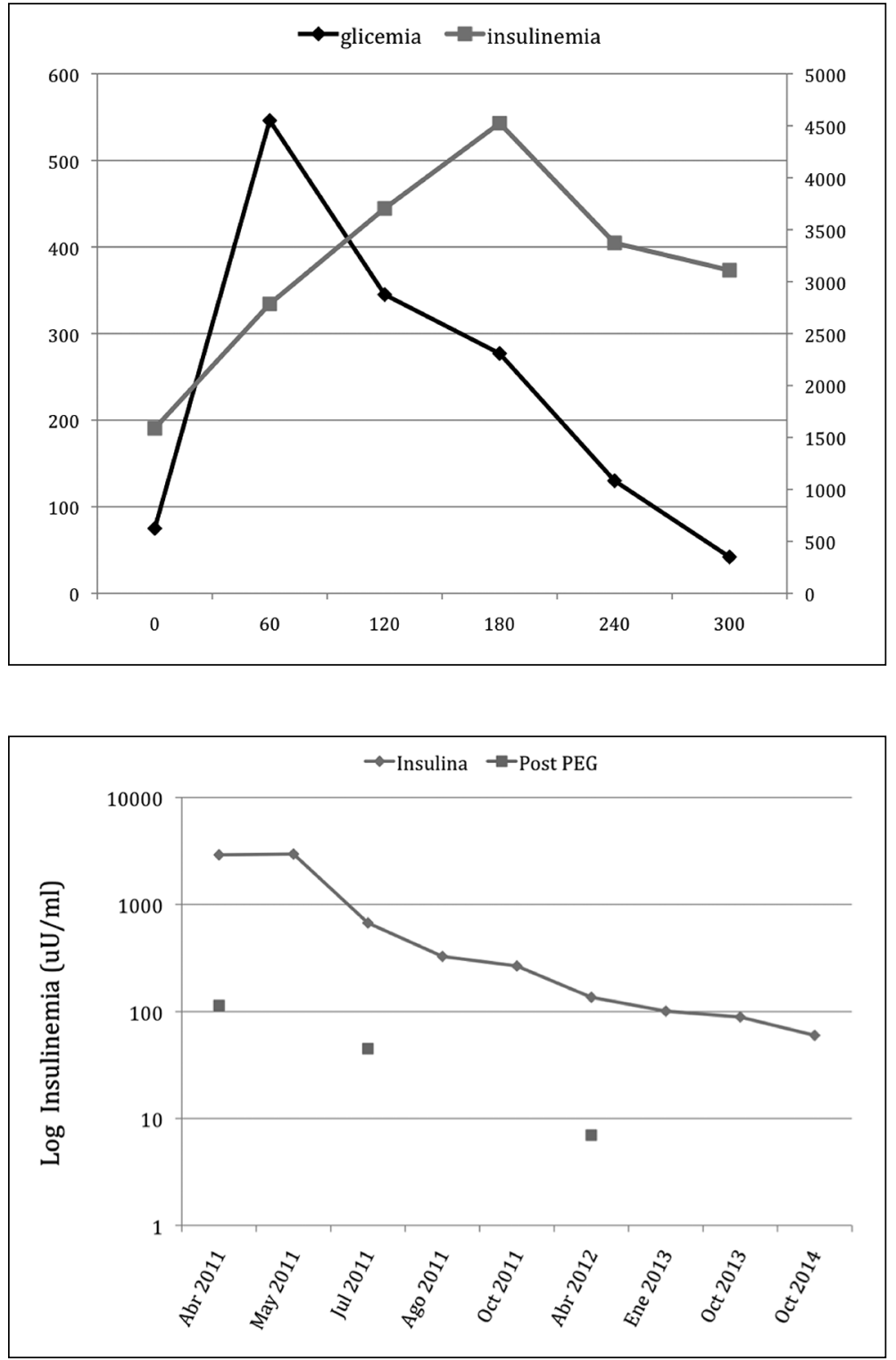

Figura 1. Prueba de tolerancia a la glucosa de 5 h con medición de insulinemia en el caso 2.
Figura 2. Evolución de niveles de Insulina total e Insulina post Polietilenglicol en el caso 2.

\section{Discusión}

El primer caso de IAS fue descrito por Hirata en 1970 y la presencia de anticuerpos a insulina endógena fue documentada en $1972^{1,4}$. Veinte años después se habían comunicado 197 casos en Japón y en más de la mitad de ellos existía el antecedente de exposición a medicamentos que poseen el grupo sulfidrilo, tales como metimazol, tiamazol, ácido alfa-lipoico, penicilamina o glutatión (Tabla $2)^{5}$. La mayoría de los casos remitieron espontá- neamente, incluso en ausencia de tratamiento, al retirar el medicamento relacionado.

Actualmente se estiman en alrededor de 300 los casos descritos en Japón.

En países occidentales la incidencia es muy baja, con comunicaciones de casos aislados en Europa $^{6-8}$ y solamente tres casos en Sudamérica ${ }^{9-11}$.

Se ha descrito una predisposición genética asociada al haplotipo HLA, siendo de mayor riesgo el haplotipo $\mathrm{DRB1}{ }^{\star} 0406, \mathrm{DQA} 1^{\star} 0301 \mathrm{y}$ $\mathrm{DQB} 1^{\star} 0302^{12}$, más frecuente en población asiática. 
Tabla 2. Condiciones asociadas al síndrome de hipoglicemia autoinmune

\begin{tabular}{|ll|}
\hline Fármacos & Patologías \\
\hline Metimazol & Enfermedad de Basedow Graves \\
\hline Propiltiuracilo & Artritis reumatoide \\
\hline Procainamida & Polimiositis \\
\hline Captopril & Urticaria crónica \\
\hline Penicilina G & Hepatopatías crónicas \\
\hline Imipenem & Pénfigo \\
\hline Hidralazina & Hipergamaglobulinemia \\
\hline Isoniazida & Leucemia mielomonocítica crónica \\
\hline Glutatión & Esclerosis sistémica \\
\hline Interferón alfa & Tuberculosis \\
\hline Esteroides & Colitis ulcerosa \\
\hline Sales de oro & \\
\hline Penicilamina & \\
\hline
\end{tabular}

En nuestro segundo caso el HLA resultó concordante con dos de los alelos descritos en estudios previos $\left(\mathrm{DQB1} 1^{\star} 0302\right.$ y DQA1* 0301). Ninguno de los pacientes tenía ancestros de origen asiático conocidos.

La hipoglicemia es generalmente reactiva y ocurre varias horas después de una comida o carga de glucosa más que en estado de ayuno, como se evidenció en nuestro segundo caso, pudiendo presentar hiperglicemia inmediatamente después de una carga de glucosa. Durante la hipoglicemia, las concentraciones de insulina, proinsulina y péptido $\mathrm{C}$ exceden considerablemente las observadas en pacientes con insulinoma y dichas mediciones están interferidas por los autoanticuerpos, los cuales tienen un porcentaje de unión a insulina humana mayor a $50 \%$ y pueden ser policlonales o monoclonales de clase IgG. El mecanismo de hiperglicemia transitoria e hipoglicemia tardía se explicaría por la teoría del efecto "buffer" de los altos niveles de anticuerpos en la biodisponibilidad de insulina en los tejidos blanco ${ }^{13}$. Esto da cuenta de la falta de respuesta en reducción de la glicemia después de la estimulación aguda de secreción de insulina, con intolerancia a la glucosa o diabetes franca, seguida de una prolongación de la vida media de la insulina la cual, al ser liberada desde el pool de anticuerpos circulantes independiente de los cambios en la glucosa plasmática, puede resultar en hipoglicemia tardía después de la ingesta de carbohidratos, pero menos probablemente después de ayuno prolongado.

También se han descrito casos de IAS asociados a mieloma múltiple, con producción de anticuerpos monoclonales por transformación desde un clon autoreactivo de células $\mathrm{B}$, que remite con el tratamiento exitoso de la neoplasia ${ }^{14}$. Por esto se ha planteado la pesquisa de paraproteinemia en casos de IAS sin una causa clara.

Debe diferenciarse de la hipoglicemia inducida por anticuerpos anti receptor de insulina, también llamada insulinoresistencia tipo B. En estos casos los anticuerpos causan hipoglicemia mediante la activación directa del receptor de insulina. Se caracteriza por la presencia de acantosis nigricans marcada, asociación con hiperglicemia severa y otras condiciones de autoinmunidad ${ }^{15}$.

Para confirmar el diagnóstico es útil la incubación de la muestra con polietilenglicol, con lo cual se logra la precipitación de los anticuerpos antiinsulina, como en nuestro segundo caso, o del péptido $\mathrm{C}$ si se encontrare elevado ${ }^{16}$.

La terapia más efectiva, además de suspender la droga causante, es ingerir varias comidas en pequeñas cantidades y evitar comidas abundantes en hidratos de carbono de alto índice glicémico. También se ha descrito de utilidad la maicena ${ }^{17}$ y los inhibidores de alfa glucosidasa como la acarbosa, que retrasan la absorción de glucosa disminuyendo la respuesta de hipersecreción de insulina ${ }^{18}$. Otra medida terapéutica utilizada son los corticoides, en forma transitoria, que a veces debe ser prolongada ${ }^{19}$. En caso de no haber respuesta se puede plantear el uso de inmunosupresores o plasmaferesis ${ }^{14}$.

Recientemente se ha planteado el uso de monitoreo continuo de glucosa para evaluar las excursiones de glicemias con distintas alternativas de terapia y objetivar su efectividad ${ }^{20}$.

En conclusión, pese a la muy baja incidencia del síndrome, se debe sospechar IAS frente a un caso de hipoglicemia espontánea en que se presentan concentraciones extremadamente elevadas de insulina. En estos casos se debe realizar medición de anticuerpos antiinsulina y tratar la muestra con polietilenglicol para precipitar los anticuerpos y determinar la insulina libre, logrando una aproximación diagnóstica oportuna, evitando estudios de imágenes de alto costo o invasivos y poder realizar una terapia adecuada. 


\section{Referencias}

1. Hirata Y, Ishizu H, Ouchi N, Motomura S, Abe M, Hara $\mathrm{Y}$, et al. Insulin autoimmunity in a case of spontaneous hypoglycemia. J Jap Diabetes Soc 1970; 13: 312-20.

2. Martínez D, Silva G, Solís I, Toloza J, Benavides C, Hamilton J, et al. Test de estimulación intraarterial selectiva con calcio en el diagnóstico de localización de insulinomas. Casos clínicos. Rev Med Chile 2004; 132: 71-4.

3. Fahie-Wilson MN, Soule SG. Macroprolactinaemia: contribution to hyperprolactinaemia in a disctrict general hospital and evaluation of a screening test based on precipitation with polyethylene glycol. Ann Clin Biochem 34 (1997), pp. 252-8.

4. Hirata Y, Ishtz H. Elevated Insulin-binding Capacity of Serum Proteins in a Case with Spontaneous Hypoglycemia and Mild Diabetes Not Treated with Insulin. Tohoku J Exp. Med 1972; 107: 277-86.

5. Uchigata Y, Eguchi Y, Takayama-Hasumi S, Omori Y. Insulin autoimmune syndrome (Hirata disease): clinical features and epidemiology in Japan. Diabetes Res Clin Pract 1994; 22 (2-3): 89-94.

6. Burch HB, Clement S, Sokol MS. Reactive hypoglycemic coma due to insulin autoimmune syndrome: case report and literature review. Am J Med 1992; 92: 681-5.

7. Cavaco B, Uchigata Y, Porto T, Amparo-Santos M, Sobrinho L, Leite V. Hypoglycaemia due to insulin autoimmune syndrome: report of two cases with characterisation of HLA alleles and insulin autoantibodies. European J Endocrinol 2001; 145: 311-6.

8. Gullo D, Evans JL, Sortino G, Goldfine ID, Vigneri R. Insulin autoimmune syndrome (Hirata Disease) in European Caucasians taking -lipoic acid. Clin Endocrinol (Oxf) 2014; 81 (2): 204-9.

9. Cresto JC, Abdenur JE, Chamoles N, Bresciani P, Ruiz M, Massa B, et al. Autoimmune hypoglycemia syndrome with specific anti-human insulin antibodies. Medicina (Buenos Aires) 1996; 56 (3): 279-83.

10. Moreira RO, Lima GA, Peixoto PC, Farias ML, Vaisman $\mathrm{M}$. Insulin autoinmune syndrome: case report. Sao Paulo Med J 2004; 122 (4): 178-80.

11. Trabucchi A, Iacono R, Guerra L, Faccinetti N, Krochik
A, Arriazu M, et al. Characterization of Insulin Antibodies by Surface Plasmon Resonance in Two Clinical Cases: Brittle Diabetes and Insulin Autoimmune Syndrome. PLoS One 2013; 8 (12): e84099.

12. Uchigata Y, Kuwata S, Tokunaga K, Eguchi Y, Takayama-Hasumi S, Miyamoto M, et al. Strong association of insulin autoimmune syndrome with HLA-DR4. Lancet 1992; 339 (8790): 393-4.

13. Dozio N, Scavini M, Beretta A, Sarugeri E, Sartori $\mathrm{S}$, Belloni $\mathrm{C}$, et al. Imaging of the Buffering Effect of Insulin Antibodies in the Autoimmune Hypoglycemic Syndrome. J Clin Endocrinol Metab 1998; 83: 643-8.

14. Waldron-Lynch F, Inzucchi SE, Menard L, Tai N, Preston-Hurlburt $\mathrm{P}$, Hui $\mathrm{P}$, et al. Relapsing and remitting severe hypoglycemia due to a monoclonal anti-insulin antibody heralding a case of multiple myeloma. J Clin Endocrinol Metab 2012; 97 (12): 4317-23.

15. Lupsa B, Chong A, Cochran E, Soos M, Semple R, Gorden P. Autoimmune Forms of Hypoglycemia. Medicine 2009; 88: 141-53.

16. Arzamendi AE, Rajamani U, Jialal I. Pseudoinsulinoma in a white man with autoimmune hypoglycemia due to anti-insulin antibodies: value of the free C-Peptide assay. Am J Clin Pathol 2014; 142 (5): 689-93.

17. Deguchi A, Okauchi Y, Suehara S, Mineo I. Insulin autoimmune syndrome in a health supplement user: the effectiveness of cornstarch therapy for treating hypoglycemia. Intern Med 2013; 52 (3): 369-72.

18. Nasu T, Suzuki R, Okamoto Y, Miyata K, Uno A, Nakao R. Late Postprandial Hypoglycemia due to Bioactive Insulin Dissociation from Autoantibody Leading to Unconsciousness in a Patient with Insulin Autoimmune Syndrome. Intern Med 2011; 50: 339-43.

19. Gómez Cruz M, Jabbar M, Saini N, Eng D, Crawford B, Vásquez D, et al. Severe hypoglycemia secondary to methimazole-induced insulin autoimmune syndrome in a 16 year old African-American male. Pediatric Diabetes 2012; 13 (8): 652-65.

20. Philippon M, Sejil S, Mugnier M, Rocher L, Guibergia C, Vialettes B, et al. Use of the continuous glucose monitoring system to treat insulin autoimmune syndrome: quantification of glucose excursions and evaluation of treatment efficacy. Diabet Med 2014; 31 (7): e20-4. 